\title{
Exploding Offers and Unraveling in Two-Sided Matching Markets
}

\author{
Siqi Pan*
}

July 1, 2017

\begin{abstract}
Many two-sided matching markets tend to unravel in time with transactions becoming inefficiently early. In a two-period decentralized model, this paper shows that when a market culture allows firms to make exploding offers, unraveling is more likely to occur and lead to a less socially desirable matching outcome. A market with a larger uncertainty in early stages is not necessarily more vulnerable to the presence of exploding offers: the conclusion depends on the specific information structure. A market tends to be less vulnerable to exploding offers when there is an excess supply of labor. While a banning policy on exploding offers tends to be supported by high quality firms and workers, it can be opposed by those of low qualities. This explains the prevalence of exploding offers in practice.
\end{abstract}

\section{Introduction}

Many two-sided matching markets exhibit the tendency to unravel in time with transactions occurring earlier and earlier. In these markets, with participants' qualities gradually revealed over time, early transactions can lead to significant efficiency losses: due to the lack of information in early stages, a higher probability of mismatch often leads to instability and thus costly rematching procedures afterwards. ${ }^{1}$ In order to effectively halt such an unraveling process, the previous literature has identified several factors that may influence early moving incentives; one of them is the use of exploding offers.

${ }^{*}$ Department of Economics, The University of Melbourne, Victoria, 3010 Australia (e-mail: siqi.pan@unimelb.edu.au). I would like to thank Paul J. Healy, Yaron Azrieli, Clayton Featherstone, Alex Gotthard-Real, John Kagel, James Peck, Garrett Senney, Wing Suen, Bruce Weinberg, Huanxing Yang, Lixin Ye, seminar participants at the Ohio State University, and audiences at Midwest Economic Theory Conference for helpful comments and inspiration. All remaining errors are mine.

${ }^{1}$ Roth and Xing (1994) provide a detailed overview of various evidence for market unraveling. See also Mongell and Roth (1991), Haruvy, Roth, and Ünver (2006), Avery, Fairbanks, and Zeckhauser (2009), Avery et al. (2001), and Fréchette, Roth, and Ünver (2007). 
An exploding offer is an offer that comes with a time limit. The offer has to be accepted within the time limit, or it is considered rejected. In contrast, an open offer can be held until the end of the market. According to Niederle and Roth (2009), exploding offers are prevalently used in many markets facing serious unraveling problems, such as the market for new gastroenterologists in the US. In a lab experiment, they are able to reproduce the facilitating effect of exploding offers on early transactions. However, not all markets that use exploding offers suffer from unraveling. For example, in the job market for junior economists, offers often come with short time limits, yet little tendency towards unraveling has been observed so far.

This paper aims to reconcile the above phenomena from a theoretical perspective. In a twoperiod decentralized matching market, the true qualities of workers are not fully revealed until the second period; firms and workers only observe a signal in the first period. By comparing markets with or without a banning policy on exploding offers, I show that when firms are allowed to make exploding offers, equilibria without unraveling are supported by a smaller parameter space and thus a stable matching is less likely to be achieved. Intuitively, a worker tends to exploit an open offer by holding it until the last period. In response, a firm uses an exploding offer to eliminate the risk of being rejected and remaining unmatched at end of the market. The time limit forces the worker to balance the cost of rejecting the current offer and the likelihood of receiving a better offer in the future. As a result, an early exploding offer is more easily accepted than an early open offer, and thus the market is more likely to unravel when exploding offers are allowed. In addition, I identify the sufficient conditions under which an equilibrium without unraveling never exists, and the sufficient and necessary condition for the market to fully unravel.

The above results indicate that the use of exploding offers is only a necessary but not a sufficient condition for unraveling to occur. An important question we should ask is what characteristics of a market can make it less likely to be affected by exploding offers? This study highlights two findings on comparative statics. First, signal accuracy has an ambiguous effect on how vulnerable a market is to exploding offers; the conclusion depends on the specific information structure. As mentioned above, in the first period, market participants only observe a signal suggesting each worker's potential quality or type. As the signal becomes more accurate, a firm is more willing to make an early exploding offer to a high type worker while the worker is less willing to accept; a firm is less willing to make an early exploding offer to a low type worker while the worker is more willing to accept. In other words, the early moving incentives always change in opposite directions for different sides of the market. The overall effect of signal accuracy hinges on how uncertainty is resolved in a specific market. Under the same framework, I show that opposite effects can be produced simply by altering the information structure of the market.

Such a finding reconciles the different conclusions from two previous studies. Roth and 
Xing (1994) find that unraveling tends to be impeded if the uncertainty in early stages is sufficiently large; yet a non-monotonic relationship is shown in Fainmesser (2013): an increase in signal accuracy generates greater market unraveling when the signal is inaccurate enough, while the effect reverses when the signal is sufficiently accurate. This paper suggests that the information structure is a key element that can affect the comparative statics on signal accuracy.

Second, a market tends to be less vulnerable to exploding offers when there is an excess supply of labor, that is, when workers outnumber job vacancies in the market. In this case, low quality workers remain unmatched in an equilibrium without unraveling, and a firm incurs the risk of hiring these workers by moving before the resolution of uncertainty. Therefore, the firm may be unwilling to deviate even when an early exploding offer would be accepted.

Given the facilitating effect of exploding offers on early transactions, a natural policy consideration is whether exploding offers should be allowed in a market. I investigate the welfare aspect by asking the following questions. Suppose there is a proposal on a ban towards exploding offers. Who would support? Who would oppose? The result indicates that such a banning policy can be supported by high quality firms and workers but opposed by agents of lower qualities. This provides a theoretical support for the experimental findings in Niederle and Roth (2009). In their exploding offer treatment, some higher (lower) quality firms and workers receive significantly lower (higher) payoffs than in the open offer treatment. The conclusion also explains the prevalence of exploding offers in practice and why it is sometimes difficult to achieve consensus on how to solve the unraveling problem in real markets. ${ }^{2}$

\section{$1.1 \quad$ Related Literature}

This paper provides a theoretical analysis on several market characteristics that could affect market unraveling: the use of exploding offers, signal accuracy, and labor demand and supply. Using an experimental approach, Niederle and Roth (2009) show that exploding offers has a facilitating effect on early transactions. In a social network model, Fainmesser (2013) also shows that the use of exploding offers is a necessary condition for unraveling to occur.

To the best of our knowledge, this paper is the first to identify information structure as a key element that can affect the comparative statics on signal accuracy, thus reconciling different conclusions from the previous studies. Roth and Xing (1994) find that a more accurate signal tends to impede market unraveling. However, Fainmesser (2013) finds a non-monotonic relationship between signal accuracy and the unraveling level in the market: moderate levels of accuracy generate greater unraveling than high or low extreme levels.

This paper studies the effect of labor demand and supply imbalances while keeping a linear quality distribution over participants on each side. Labor demand and supply are also

\footnotetext{
${ }^{2}$ For example, see a discussion regarding the US market for new gastroenterologists by Niederle and Roth (2005).
} 
investigated by Niederle, Roth, and Ünver (2013) using an experimental approach. However, they mainly focus on the effect of altering quality distributions. With two possible qualities on each side, they conclude that unraveling can only occur when there is comparable demand and supply: a surplus of workers, but a shortage of high quality workers. In a specific environment that involves both decentralized and centralized settings, Niederle and Roth (2003) suggest that the unraveling in the gastroenterology market might have been triggered by a demand shock. McKinney, Niederle, and Roth (2003) conduct an experimental study on demand and supply imbalances tailored to this market.

Besides the market characteristics discussed above, the previous literature has identified several other factors that could affect unraveling, including the stability of centralized matching algorithms (Roth, 1984, 1991; Kagel and Roth, 2000; Ünver, 2001, 2005), market congestion (Roth and Xing, 1997), similarity of preferences (Hałaburda, 2010), social network structures (Fainmesser, 2013), and strategic complementarities (Echenique and Pereyra, 2016). The motives behind unraveling are also investigated in some different environments. Under the framework of competitive markets, Li and Rosen (1998), Li and Suen (2000, 2004), and Suen (2000) show how unraveling can occur as a form of insurance in the absence of complete markets. In a model with asymmetric information, but without evolving uncertainty, Lee (2009) explains early contacting as a way to avoid adverse selection.

\section{The Model}

Consider a two-sided matching market with $F$ firms and $W$ workers, where $F \geq 3$ and $W \geq 3$. Let $\mathcal{F}=\left\{f_{1}, \ldots, f_{F}\right\}$ be the set of firms, and $\mathcal{W}=\left\{w_{1}, \ldots, w_{W}\right\}$ be the set of workers. Each firm has the capacity to hire at most one worker, and each worker can work for at most one firm. A market with $W<F$ (or $W>F$ ) is said to have excess demand (or excess supply) of labor.

All workers agree on the same ranking of firms: $f_{F} \succ f_{F-1} \succ \ldots \succ f_{1}$, and all firms agree on the same ranking of workers: $w_{W} \succ w_{W-1} \succ \ldots \succ w_{1}{ }^{3}$ The ranking of firms is common knowledge to the entire market. The true ranking of workers is revealed over time. Let $\mathcal{R}$ be the set of all possible strict rankings of workers, in which each ranking/state is realized with equal probability $\frac{1}{W !}$. Denote the true ranking/state as $\succ \in \mathcal{R}$.

In terms of utility, all firms value a match with the $i$-th ranked worker in the true state $\left(w_{i}\right)$ as $v_{i}=i$, and all workers value a match with the $j$-th ranked firm $\left(f_{j}\right)$ as $u_{j}=j$. Unmatched market participants derive zero utility: $v_{0}=u_{0}=0$. Therefore, any match is preferable to remaining unmatched. Notice in this setting, a firm's utility from a match depends only on

\footnotetext{
${ }^{3}$ Clearly, this is a simplifying assumption. The similarity of preferences is also a factor that can affect market unraveling. Hałaburda (2010) considers the similarity of firms' preferences over workers as a comparative statics parameter while having all workers agree on the same ranking of firms. The result shows that similarity of preferences can drive unraveling.
} 
the worker's rank, and a worker's utility depends only on the firm's rank. The two functions $v_{i}$ and $u_{j}$ indicate worker quality and firm quality respectively. ${ }^{4}$

The outcome of a matching market, that is, a matching is said to be stable if and only if there is no worker-firm pair in which each prefers one another to her current match. Since the existence of such pairs often leads to costly rematching procedures afterwards, stability is used as a central criterion to evaluate market outcomes by the two-sided matching literature. In the current environment with strict rankings and aligned preferences, it is easy to see that the assortative matching in the true state $\succ$ constitutes the unique stable matching.

The market lasts for two hiring periods, with the true ranking $\succ$ revealed in Period 2. At the beginning of Period 1, a public signal $\hat{\succ} \in \mathcal{R}$ is observed by both firms and workers. With probability $\alpha, \hat{\succ}$ is the same as $\succ$. Otherwise, $\hat{\succ}$ is a uniform random draw from $\mathcal{R}$. The parameter $\alpha \in(0,1)$ measures signal accuracy: a larger value of $\alpha$ indicates a smaller uncertainty faced by the market. Denote a worker's type in Period 1 as $\hat{r}$, which is her rank in $\hat{\succ}$. Denote a worker's true rank as $r$, which is her rank in $\succ$.

The game proceeds as follows. In Period 1, a public signal $\hat{\succ}$ is observed. Next, each firm simultaneously makes an offer to at most one worker. Finally, each worker simultaneously chooses at most one offer to accept from those available to her. A similar procedure takes place in the second period, except that the true ranking of workers $\succ$, instead of a signal, is observed at the beginning of the period. All actions of firms and workers are publicly observed.

I focus my discussion on two types of offers: exploding offers and open offers.

Definition 1. An exploding offer is an offer that comes with a time limit. It can only be accepted within the time limit. Otherwise, it is rejected.

Definition 2. An open offer is an offer that can be held until the last period.

In the current two-period model, an exploding offer has to be accepted immediately, in the same period in which it is made. However, if a worker receives an open offer in the first period, she could choose to hold it until Period 2. An open offer made in Period 2 is equivalent to an exploding offer.

Regarding the culture or norms of the market, I make the following two assumptions.

\footnotetext{
${ }^{4}$ With a linear quality distribution, the main results of the paper do not hinge on a specific quality range. However, some results may not hold for other quality distributions. Quality distribution is also a factor that can affect market unraveling. An example that allows non-linear quality distributions can be found in the working paper version of this paper (https://www.dropbox.com/s/xtb9zv5tuuyhi4e/Exploding_Offers_WorkingPaper0801.pdf?dl=0). It suggests that a market is less likely to be affected by exploding offers if the quality distribution over firms is more convex, or the quality distribution over workers is more concave. With two possible qualities on each side of the market, Niederle, Roth, and Ünver (2013) show in a lab experiment that unraveling only occurs when demand and supply are comparable, that is, when there exist excess workers, but a shortage of high quality workers.
} 
Assumption 1. (Binding acceptances) Once a worker accepts an offer, the acceptance is binding. A worker cannot renege on her acceptance.

Assumption 2. (Binding rejections) Once a worker rejects an offer, the rejection is binding. A firm will not make an offer to the same worker again.

Following Niederle and Roth (2009), Assumption 1 is made to ensure the validity of exploding offers. Assumption 2 is an important and reasonable addition because (i) it reflects the norms of some real-life two-sided matching markets such as the market for judicial clerks; (ii) it increases the power of exploding offers by raising workers' rejection costs. Hence, without such an assumption, the effects of exploding offers on market outcomes can be largely underestimated. ${ }^{5}$

\section{Equilibrium Analysis}

In this section, I start with a baseline case where firms can only make open offers, and then relax the constraint by allowing exploding offers. The discussion mainly concerns two types of subgame perfect Nash equilibria in weakly undominated pure strategies: those without unraveling, and those with full unraveling.

The following characterization of equilibria focuses on the timing of offers made by "relevant" firms, which include all firms as $W \geq F$ and only firms $f_{F}, f_{F-1}, \ldots$, and $f_{F-W+1}$ as $W<F$. This is because in either type of equilibria, $f_{F-W}, f_{F-W-1}, \ldots$, and $f_{1}$ as $W<F$ are indifferent among all possible strategies and their actions do not affect the equilibrium outcome.

Definition 3. An equilibrium without unraveling is an equilibrium where no relevant firms make any offers until the last period.

In an equilibrium without unraveling, no actions are taken by relevant firms in Period 1. In Period 2, two cases are considered separately: (i) when $W \geq F, f_{F}$ makes an offer to $w_{W}$, $f_{F-1}$ to $w_{W-1}, \ldots, f_{1}$ to $w_{W-F+1}$, and all offers are accepted; (ii) when $W<F, f_{F}$ makes an offer to $w_{W}, f_{F-1}$ to $w_{W-1}, \ldots$, and $f_{F-W+1}$ to $w_{1}$, and all these offers are accepted.

Definition 4. An equilibrium with full unraveling is an equilibrium where every relevant firm makes an early offer in Period 1.

\footnotetext{
${ }^{5}$ The reality in some markets is less stringent than Assumption 2. For example, in the job market for junior economists, although when rejecting an exploding offer, a candidate typically does not consider the possibility that she may receive an offer from the same employer again, the phenomenon of nonbinding rejections is still observed in some situations. In this case, a more realistic setting is to have each firm decide whether to raise its leverage by attaching a commitment of binding rejection when making an early exploding offer. For these markets, although Assumption 2 significantly simplifies the analysis, it can lead to an overestimation of the effects of exploding offers.
} 
In an equilibrium with full unraveling, after $\hat{\succ}$ is revealed in Period 1 , two cases are considered separately: (i) when $W \geq F, f_{F}$ makes an offer to type $\hat{r}=W, f_{F-1}$ to $\hat{r}=W-1$, ..., $f_{1}$ to $\hat{r}=W-F+1$, and all offers are accepted; (ii) when $W<F, f_{F}$ makes an offer to type $\hat{r}=W, f_{F-1}$ to $\hat{r}=W-1, \ldots, f_{F-W+1}$ to $\hat{r}=1$, and all these offers are accepted.

In both types of equilibria, when $W<F$, firms $f_{F-W}, f_{F-W-1}, \ldots$, and $f_{1}$ may adopt any strategy; their offers (if made) are not accepted by any workers. Clearly, the unique outcome of equilibria without unraveling is the assortative matching according to the true ranking of workers $\succ$, which is the unique stable matching in the current setting. The unique outcome of equilibria with full unraveling is the assortative matching according to the signal-suggested ranking of workers $\hat{\succ}$, which is only stable when the signal is correct.

In addition, an equilibrium is said to have partial unraveling if some relevant firms make offers in Period 1, and some make offers in Period 2.

\subsection{Open Offers Only}

Consider the case where firms can only make open offers due to the culture, norms, or policies in a market environment. ${ }^{6}$ The following proposition describes the equilibrium outcome.

Proposition 1. When firms are not allowed to make exploding offers, there only exist equilibria without unraveling; the stable matching is the unique equilibrium outcome.

While the full proof is provided in the appendix, the basic intuition is clear. In Period 1, every type of worker has a positive probability of having the highest quality in the true state. Therefore, as long as the best firm $f_{F}$ moves in Period 2, a worker strictly prefers to hold any early open offer until Period 2. Knowing this, $f_{F}$ strictly prefers to wait until the last period, so that all workers will stay in the market and the one of the highest quality can be perfectly identified. Since no offer is accepted in Period 1, the other firms cannot make themselves better off by moving early; instead, they incur the risk of being rejected in the last period and remaining unmatched.

Proposition 1 shows that in a market where firms only use open offers, an equilibrium without unraveling always exists, while an equilibrium with full unraveling never does. This provides us with a very clean baseline, so that the effects of exploding offers can be easily identified from the change in the parameter spaces supporting these two types of equilibria.

\subsection{Exploding and Open Offers}

Now I consider the case where both open offers and exploding offers can be made in a market.

\footnotetext{
${ }^{6}$ For example, exploding offers are publicly discouraged in the US market for new graduate students. The Council of Graduate Schools has published a resolution stating that students are under no obligation to respond to offers of financial support prior to April 15 (http://cgsnet.org/april-15-resolution).
} 
Lemma 1. In an equilibrium in undominated strategies, firms $f_{F-1}, f_{F-2}, \ldots$, and $f_{1}$ never make an open offer in Period 1 when they are allowed to make exploding offers.

The best firm $f_{F}$ is indifferent between an open offer and an exploding offer since neither of them will be rejected by any worker. In Period 2, every firm is indifferent because an open offer is equivalent to an exploding offer. Hence, in the subsequent analysis of equilibria in weakly undominated strategies, Lemma 1 allows us to consider only exploding offers without loss of generality.

After observing a signal $\hat{\succ}$ in Period 1 , both firms and workers update their beliefs. Posteriors on the true state $\succ$ are given by

$$
\operatorname{Pr}(\hat{\succ} \mid \hat{\succ})=\alpha+\frac{1-\alpha}{W !},
$$

and

$$
\operatorname{Pr}\left(\succ^{\prime} \mid \hat{\succ}\right)=\frac{1-\alpha}{W !}, \forall \succ^{\prime} \neq \hat{\succ} .
$$

Posteriors on the true rank $r$ of a type- $\hat{r}$ worker are given by

$$
\operatorname{Pr}(\hat{r} \mid \hat{r})=\alpha+\frac{1-\alpha}{W}
$$

and

$$
\operatorname{Pr}\left(r^{\prime} \mid \hat{r}\right)=\frac{1-\alpha}{W}, \forall r^{\prime} \neq \hat{r}
$$

The posteriors described above involve a spike at the point $\hat{\succ}$, which indicates a high probability of the true state being the signal-suggested ranking, and an equally low probability of any other ranking being realized. ${ }^{7}$ Therefore, the assortative pairs in the state $\hat{\succ}$ will be frequently used in the subsequent analysis. These "signal-suggested pairs" are formally defined as follows.

Definition 5. The signal-suggested type of a firm $f_{j}$ is a function defined as

$$
\hat{r}(j) \equiv \begin{cases}j+W-F & \text { if } j>F-W \\ 0 & \text { if } j \leq F-W .\end{cases}
$$

The signal-suggested firm of a type $\hat{r}$ is a function defined as

$$
j(\hat{r}) \equiv \begin{cases}\hat{r}-W+F & \text { if } \hat{r}>W-F \\ 0 & \text { if } \hat{r} \leq W-F\end{cases}
$$

\footnotetext{
${ }^{7}$ In Section 3.3, I provide an example of a different information structure, under which the probability of a ranking gradually decreases as its Kendall $\tau$ distance to the signal-suggested ranking increases.
} 
A firm and its signal-suggested type, or a type of worker and its signal-suggested firm, are called a signal-suggested pair.

\subsubsection{Equilibria without Unraveling}

As mentioned in the introduction, in practice not all markets that use exploding offers suffer from unraveling. Proposition 2 provides sufficient conditions for an equilibrium without unraveling to sustain even when exploding offers are allowed in a market, while Proposition 3 provides sufficient conditions for such an equilibrium never to exist.

Recall a market with $W<F$ (or $W>F$ ) is said to have excess demand (or excess supply) of labor. I further identify the case of extreme excess supply if $W \geq 2 F$, and the case of moderate excess supply if $F<W \leq 2 F-1$.

Proposition 2. When firms are allowed to make exploding offers, an equilibrium without unraveling always exists if

(i) the market has an extreme excess supply of labor; or

(ii) the market has a moderate excess supply of labor and a sufficiently inaccurate signal.

Mathematically speaking, an equilibrium without unraveling always exists if (i) $W \geq 2 F$, or (ii) $F+2<W \leq 2 F-1$ and $\alpha \leq \frac{(W-F)^{2}-W+F-2}{(W-F)^{2}+W+F-2}$. A deviation from such an equilibrium involves both sides of the market: a firm should want to make an early exploding offer in Period 1 to a worker who wants to accept it. Hence, the condition for an equilibrium without unraveling to sustain requires that, for each worker type $\hat{r}$, the firms whose offers would be accepted should not be willing to offer. In most cases, the signal-suggested firm-worker pairs have the strongest incentive to deviate, thus affecting the binding constraint that drives the results in Proposition 2. Below I use an example to explain why.

Example 1. In a market with 3 firms and 3 workers, an equilibrium without unraveling yields the following assortative matching:

$\begin{array}{ccc}w_{3} & w_{2} & w_{1} \\ f_{3} & f_{2} & f_{1}\end{array}$

Consider a deviation between $f_{2}$ and a worker of type $\hat{r}=3$. Given the posteriors on this type in Period 1, the offer will be accepted if $2 \geq\left(\alpha+\frac{1-\alpha}{3}\right) \times 3+\frac{1-\alpha}{3} \times 1+\frac{1-\alpha}{3} \times 1$. While the LHS gives the worker's utility when accepting the offer, the RHS is the worker's expected utility when rejecting. The second part of the RHS indicates a rejection cost of $\frac{1-\alpha}{3}$ : after rejecting $f_{2}$, the worker can only receive an offer from $f_{1}$ even when her true rank turns out to be $r=2$ in Period 2. On the other hand, if $f_{2}$ makes an early offer to its signal-suggested type $\hat{r}=2$, the offer will be accepted when $2 \geq \frac{1-\alpha}{3} \times 3+\left(\alpha+\frac{1-\alpha}{3}\right) \times 1+\frac{1-\alpha}{3} \times 1$. Again, the second part of the RHS indicates a rejection cost of $\left(\alpha+\frac{1-\alpha}{3}\right)$. 
Example 1 shows that a deviation between a signal-suggested pair tends to succeed more easily: although a firm always prefers higher types, its signal-suggested type is more likely to accept its early offer due to the higher rejection cost $\left(\alpha+\frac{1-\alpha}{3}>\frac{1-\alpha}{3}\right)$. This is quite intuitive since it indicates that a worker is more reluctant to reject what appears to be "a good match" in Period 1 - a firm that is most likely to be her match in a stable matching. Therefore, for an equilibrium without unraveling to sustain, the binding condition in most cases requires the best firm that would be accepted by its corresponding type not be willing to offer.

Condition (ii) of Proposition 2 is mainly driven by the workers' side of the market. As the signal in Period 1 becomes less accurate, the cost of rejecting a signal-suggested firm $\left(\alpha+\frac{1-\alpha}{3}\right)$, or more generally, $\left(\alpha+\frac{1-\alpha}{W}\right)$ decreases. Thus, a worker is more likely to reject an exploding offer and an equilibrium without unraveling is more likely to sustain. ${ }^{8}$ On the other hand, condition (i) stems from a boundary solution on the firms' side, in which case no firm is willing to make an early offer to its signal-suggested type even if it is always accepted. It arises when there is an extreme excess supply of labor $(W \geq 2 F)$, which means in an equilibrium without unraveling, even the worst firm $f_{1}$ is matched with an above-average worker. This significantly increases a firm's risk in making an offer before the resolution of uncertainty since the worker is more likely to have a lower quality compared to the firm's match in equilibrium.

Proposition 3. When firms are allowed to make exploding offers, an equilibrium without unraveling never exists if

(i) the market has an excess demand of labor and a sufficiently accurate signal; or

(ii) the market has a moderate excess supply of labor and a sufficiently accurate signal.

Mathematically speaking, an equilibrium without unraveling never exists if (i) $W \leq F$ and $\alpha>\frac{W-1}{2 W-1}$, or (ii) $F<W<2 F-1$ and $\alpha>\frac{(W-F)^{2}+W+F-2}{(W-F)^{2}+3 W+F-2}$. For a deviation to occur, there must exist a type of worker and a firm in Period 1 such that, the firm is willing to offer and the worker is willing to accept. Again, the binding condition here hinges on the deviations between signal-suggested pairs. Proposition 3 shares the similar intuition with Proposition 2: a more accurate signal encourages a deviation by increasing the worker's rejection cost, while an excess demand of labor decreases the firm's risk of being worse off in a deviation.

\footnotetext{
${ }^{8}$ Although the cost of rejecting a non-signal-suggested firm $\frac{1-\alpha}{W}$ increases as the signal becomes less accurate, here the elimination of such a deviation is not the binding condition and does not drive the results. In Section 3.3, I provide an example with a different information structure, under which the non-signal-suggested pairs can also influence the binding condition, and thus signal accuracy may have the opposite effect on market unraveling.
} 


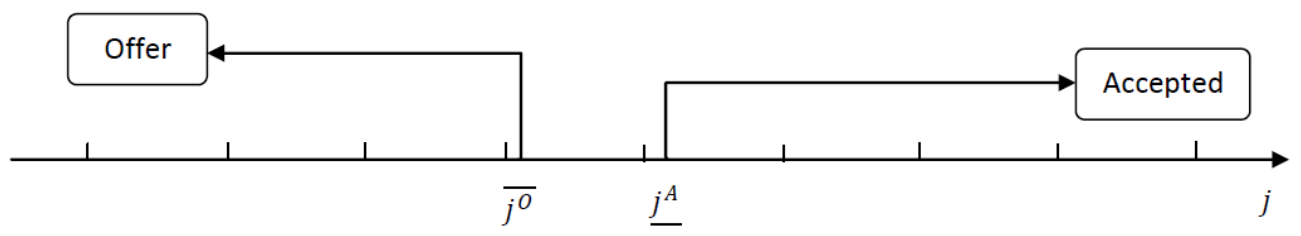

(a) A Sufficient Condition for the Existence of an Equilibrium Without Unraveling

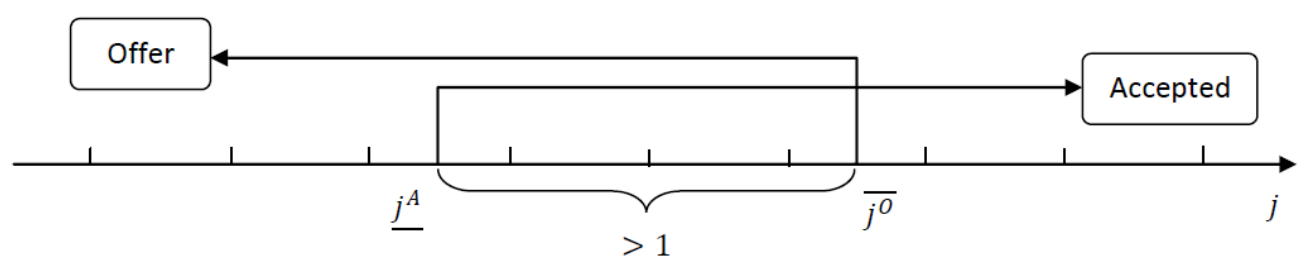

(b) A Sufficient Condition for the Non-existence of an Equilibrium Without Unraveling

Figure 1: An Integer Problem

There exists a gap between the two parameter spaces identified in Propositions 2 and 3. Let $\alpha_{1}$ and $\alpha_{2}$ be functions of $W$ and $F$ such that $\alpha_{1}(W, F) \equiv \frac{(W-F)^{2}-W+F-2}{(W-F)^{2}+W+F-2}$ and $\alpha_{2}(W, F) \equiv \frac{(W-F)^{2}+W+F-2}{(W-F)^{2}+3 W+F-2}$. For some cases of moderate excess supply $(F+2<W<2 F-1)$, an equilibrium without unraveling always exists when $\alpha \leq \alpha_{1}$ and never exists when $\alpha>\alpha_{2}$. It is easy to confirm that $\alpha_{1}<\alpha_{2}$.

Such a gap is due to an integer problem, which makes conditions in Propositions 2 and 3 sufficient but not necessary. Denote $j^{A}$ as the lowest quality firm whose early offer is accepted by its signal-suggested type and $\overline{j^{O}}$ as the highest quality firm willing to make such an offer given the acceptance. For an equilibrium without unraveling to exist, a sufficient and necessary condition only requires no integer to be in the range between $\underline{j}^{A}$ and $\overline{j^{O}}$, which depends on the specific parameter values of $\alpha, W$, and $F$. In order to draw a general conclusion, we need to have the range completely empty, that is, $\underline{j}^{A} \geq \overline{j^{O}}$ (Figure 1a). Similarly, although an equilibrium without unraveling does not exist as long as there is an integer in the range between $j^{A}$ and $\overline{j^{O}}$, for a general conclusion, we need to set the range larger than 1 , that is, $\overline{j^{O}}-\underline{j^{A}>1}$ (Figure $\left.1 \mathrm{~b}\right)$.

For cases within the gap, whether unraveling will occur depends on the specific values of $\alpha, F$, and $W$. To illustrate, I provide the following examples where $F+2<W<2 F-1$ and $\alpha \in\left(\alpha_{1}, \alpha_{2}\right]$. An equilibrium without unraveling exists in case (1) but not in case (2).

Example 2. (1) When $\alpha=0.51, F=5$, and $W=8$, there does not exist an equilibrium without unraveling: given $\underline{j^{A}} \approx 0.7$ and $\overline{j^{O}}=1.5$, we know that the signal-suggested pair $f_{1}$ and $\hat{r}=4$ has an incentive to deviate. 
(2) When $\alpha=0.51, F=7$, and $W=12$, an equilibrium without unraveling can sustain since there is no integer between $\underline{j^{A}} \approx 1.2$ and $\overline{j^{O}}=1.5$.

\subsubsection{Equilibria with Full Unraveling}

Now we discuss an extreme case, where the market fully unravels with every relevant firm making an exploding offer in Period $1 .^{9}$

Proposition 4. When firms are allowed to make exploding offers, an equilibrium with full unraveling exists if and only if $W \leq F$ and $\alpha \geq \frac{W-2}{W}$, that is, the market has an excess demand and a sufficiently accurate signal.

When $W>F$, an equilibrium with full unraveling can never sustain because after Period 1 , there are $W-F$ workers left in the market. Then every firm has an incentive to deviate by waiting, in which case it becomes the only available firm in Period 2 and its choice set is expanded by $W-F$ workers after the resolution of uncertainty. When $W \leq F$, however, a firm has no incentive to wait as long as in its deviation, no worker would reject an offer from her signal-suggested firm and become available in Period 2. With a more accurate signal, it is more costly for a worker to reject her offer in equilibrium, and thus a deviation is less likely to occur.

\subsubsection{Equilibria with Partial Unraveling}

In an equilibrium with partial unraveling, only some of the relevant firms make offers in Period 1, while the others choose to wait until Period 2. In contrast to the previous two types of equilibria, partial unraveling may take various forms. In this section, I first rule out some impossible structures of such equilibria, and then demonstrate some possibilities in examples.

Remark 1. There cannot exist an equilibrium with partial unraveling where every firm that moves early has a higher quality than all the firms that choose to wait.

For example, with three firms in a market, there cannot exist an equilibrium with $f_{3}$, or $f_{3}$ and $f_{2}$ being the only early moving firm(s). Such a structure cannot sustain because there exists a profitable deviation for every early moving firm in equilibrium: by deviating to Period 2 , it becomes the highest quality firm with an expanded choice set after the resolution of uncertainty.

Combining Propositions 3 and 4, we can identify two parameter spaces where neither an equilibrium without unraveling nor an equilibrium with full unraveling exists: (i) $F<W<$ $2 F-1$ and $\alpha>\frac{(W-F)^{2}+W+F-2}{(W-F)^{2}+3 W+F-2}$; (ii) $4 \leq W \leq F$ and $\frac{W-1}{2 W-1}<\alpha<\frac{W-2}{W}$. Only equilibria with partial unraveling may exist in these cases. However, as shown in the following example, for some values of $W, F$, and $\alpha$, there does not exist any equilibrium.

\footnotetext{
${ }^{9}$ Recall that relevant firms include all firms as $W \geq F$, and only firms $f_{F}, f_{F-1}, \ldots$, and $f_{F-W+1}$ as $W<F$.
} 
Example 3. Suppose there are 4 workers and 3 firms in the market.

(1) When $\alpha \in\left(\frac{3}{7}, \frac{5}{11}\right]$, there only exists the following equilibrium: in Period 1, $f_{2}$ makes an exploding offer to type $\hat{i}=4$ and $f_{1}$ makes an exploding offer to type $\hat{i}=2$; in Period $\mathscr{Q}, f_{3}$ makes an offer to the best worker left in the market; all offers are accepted.

(2) When $\alpha \in\left(\frac{5}{11}, \frac{1}{2}\right)$, there does not exist any equilibrium.

(3) When $\alpha \geq \frac{1}{2}$, there only exist the following two equilibria. (i) In Period 1, $f_{1}$ makes an exploding offer to type $\hat{i}=2$; in Period 2, $f_{3}$ makes an offer to the best worker left in the market and $f_{2}$ makes an offer to the second best; all offers are accepted. (ii) In Period $1, f_{2}$ makes an exploding offer to type $\hat{i}=3$ and $f_{1}$ makes an exploding offer to type $\hat{i}=2$; in Period 2, $f_{3}$ makes an offer to the best worker left in the market; all offers are accepted.

The above example provides us with some interesting intuitions. In case (1), the highest type worker $\hat{i}=4$ in Period 1 is "stolen" by an early moving firm $f_{2}$. But since the signal is not accurate enough, the best firm $f_{3}$ still prefers to wait for the resolution of uncertainty. In case (2), as the signal becomes more accurate, $\hat{i}=4$ becomes more attractive but is still willing to accept an early offer from $f_{2}$. As a result, $f_{3}$ is forced to move early as well so as to prevent such an early transaction; the equilibrium in case (1) can no longer sustain. In case (3) with an even more accurate signal, $\hat{i}=4$ is no longer willing to accept $f_{2}$ in Period 1 ; both the highest type worker and the highest quality firm choose to wait. Clearly, in the analysis of equilibria with partial unraveling, signal accuracy plays an important role in determining how or whether the early moving incentives for one firm are affected by early offers of other firms, because it tells the high quality firms whether it is worthwhile to "fight" for workers of high types.

\subsection{The Ambiguous Effect of Signal Accuracy}

According to the above analysis, exploding offers has a facilitating effect on early transactions; such an effect tends to be less salient in a market with an excess supply of labor or a less accurate signal. In this section, I provide an example to show that signal accuracy may have an opposite influence under a different information structure.

Example 4. There are three workers in the market. Recall $\mathcal{R}$ is the set of all possible strict rankings of workers. The Kendall $\tau$ distance, denoted as $K\left(\succ^{\prime}, \succ^{\prime \prime}\right)$, is a function that counts the number of pairwise disagreements between two rankings $\succ^{\prime}$ and $\succ^{\prime \prime}, \forall \succ^{\prime}, \succ^{\prime \prime} \in \mathcal{R}$. In other words, $K\left(\succ^{\prime}, \succ^{\prime \prime}\right)$ measures the distance between $\succ^{\prime}$ and $\succ^{\prime \prime}{ }^{10}$ For instance, between two rankings of three workers, $K\left(w_{1} \succ w_{2} \succ w_{3}, w_{1} \succ w_{3} \succ w_{2}\right)=1, K\left(w_{1} \succ w_{2} \succ w_{3}, w_{3} \succ\right.$ $\left.w_{1} \succ w_{2}\right)=2$, and $K\left(w_{1} \succ w_{2} \succ w_{3}, w_{3} \succ w_{2} \succ w_{1}\right)=3$.

The signal $\hat{\succ}$ in Period 1 equals a ranking $\succ^{\prime}$ with probability $\frac{1}{6}\left[1+3 \beta-2 \beta K\left(\succ^{\prime}, \succ\right)\right], \forall \succ^{\prime} \in$ $\mathcal{R}$, which is decreasing in the distance between $\succ^{\prime}$ and the true state $\succ$. The parameter

\footnotetext{
${ }^{10}$ See Kendall (1938) and Kemeny (1959).
} 
$\beta \in\left(0, \frac{1}{3}\right)$ measures signal accuracy: a larger value of $\beta$ indicates a smaller uncertainty faced by the market. The posteriors are thus given by

$$
\operatorname{Pr}\left(\succ=\succ^{\prime} \mid \hat{\succ}\right)=\frac{1}{6}\left[1+3 \beta-2 \beta K\left(\succ^{\prime}, \hat{\succ}\right)\right] \forall \succ^{\prime} \in \mathcal{R} .
$$

That is, the probability of a ranking being the true state gradually decreases as its distance to the signal $\hat{\succ}$ increases. This is different from the single spike at $\hat{\succ}$ in the posteriors described in Section 3.2.

Consider the simple case with three firms. An equilibrium without unraveling exists if and only if $\beta \geq \frac{1}{4}$. This result stems from the binding condition that prevents a worker of type $\hat{i}=3$ from accepting $f_{2}$ in Period 1 , with $f_{2}$ always willing to make such an exploding offer if accepted. In contrast to Proposition 2, here the condition puts a lower bound to signal accuracy, that is, it requires the signal to be sufficiently accurate for the market not to unravel.

The intuition lies in the differences between the two information structures. The posteriors described in Section 3.2 put a high probability on the signal-suggested ranking, and an equally low probability on any other ranking. As a result, the binding condition for no unraveling mainly concerns the deviations between signal-suggested pairs. In such a pair, a more accurate signal increases the worker's rejection cost without affecting the firm's early moving incentive (given the worker's acceptance of its early exploding offer). ${ }^{11}$ Overall, a more accurate signal makes the market more vulnerable to the use of exploding offers. Under the information structure in Example 4, however, non-signal-suggested pairs can also influence the binding condition. For these pairs, as the signal becomes more accurate, a high type worker in Period 1 becomes more attractive; firms are more willing to make an early exploding offer while the worker is less willing to accept. Meanwhile, a low type worker becomes less attractive; firms are less willing to offer while the worker is more willing to accept. In other words, the early moving incentives always change in opposite directions for different sides of the market. Then in certain cases such as Example 4, when the binding condition prevents a high type worker from accepting a non-signal-suggested firm, the existence of an equilibrium without unraveling requires the signal to be sufficiently accurate. In contrast to the predictions in Section 3.2.1, here a more accurate signal can make a market less likely to be affected by exploding offers.

The above analysis suggests that the effect of signal accuracy may hinge on how uncertainty is resolved in a specific market. Such a finding reconciles the different conclusions from two previous studies. Roth and Xing (1994) find that unraveling tends to be impeded if the uncertainty in early stages is sufficiently large; yet a non-monotonic relationship is shown in Fainmesser (2013): an increase in signal accuracy generates greater market unraveling

\footnotetext{
${ }^{11}$ See equations (14) and (16) in the appendix for the expressions of $\underline{j}^{A}$ (the lowest quality firm whose early offer is accepted by its signal-suggested type) and $\overline{j^{O}}$ (the highest quality firm willing to make such an offer given the acceptance) respectively. Signal accuracy $\alpha$ only enters the former but not the latter.
} 
when the signal is inaccurate enough, while the effect reverses when the signal is sufficiently accurate. This paper suggests that the information structure is a key element that can affect the comparative statics on signal accuracy. ${ }^{12}$

\section{Welfare Analysis}

Knowing that the use of exploding offers tends to facilitate unraveling and that unraveling hurts the stability of two-sided matching markets, a natural policy consideration is whether a market should allow firms to make exploding offers. In this part, I investigate the welfare aspect by asking the following questions. Suppose there is a proposal on a ban towards exploding offers. Who would support? Who would oppose? In Section 3, I have identified two types of equilibria that are of the most interest in this environment: those without unraveling, and those with full unraveling. A comparison between these two types of equilibria can shed some light on these questions.

Suppose $W, F$, and $\alpha$ are such that $4 \leq W \leq F$ and $\alpha \geq \frac{W-2}{W}$. According to previous results, when exploding offers are banned from the market, the market does not unravel (Proposition 1); when exploding offers are allowed, the market always unravels (Proposition 3) and an equilibrium with full unraveling exists (Proposition 4).

Comparing to the assortative matching, $f_{j}$ is better off in a fully unraveled market if

$$
j+W-F<\alpha(j+W-F)+\frac{(1-\alpha)(W+1)}{2},
$$

where $j=F-W+1, F-W+2, \ldots, F .^{13}$ That is,

$$
W-F+1 \leq j<\hat{j} \equiv \frac{2 F-W+1}{2} .
$$

From $W \leq F$, we have

$$
\hat{j} \geq \frac{F+1}{2} .
$$

The LHS of (1) is $f_{j}$ 's payoff in an equilibrium without unraveling, since it is matched with worker $w_{j+W-F}$. In an equilibrium with full unraveling, $f_{j}$ is matched with its signal-suggested type $\hat{r}(j)=j+W-F$. The RHS gives the expected payoff. The function $\hat{j}$ is defined as the cutoff firm. ${ }^{14}$ Firms ranked lower than $\hat{j}$ are better off (or indifferent) in full unraveling, while those ranked higher are worse off. Inequality (3) indicates that full unraveling tends to benefit medium or low quality firms while hurting high quality firms.

\footnotetext{
${ }^{12}$ Admittedly, this paper only provides an example where information structure could alter the comparative statics on signal accuracy. It is not to say that such a conclusion will hold for every market environment.

${ }^{13}$ Firms $f_{1}, f_{2}, \ldots, f_{F-W}$ are indifferent since they are unmatched in both cases.

${ }^{14}$ There is an abuse of language here since $\hat{j}$ is not necessarily an integer.
} 
On the workers' side, $w_{i}$ is better off in full unraveling if

$$
i-W+F<\alpha(i-W+F)+(1-\alpha)\left[\frac{(W+1)}{2}-W+F\right]
$$

or equivalently,

$$
i<\hat{i} \equiv \frac{W+1}{2} .
$$

The LHS of (4) is $w_{i}$ 's payoff in an equilibrium without unraveling, since it is matched with firm $f_{i-W+F}$. In an equilibrium with full unraveling, $w_{i}$ is matched with firm $j(\hat{r})=\hat{r}-W+F$, which depends on her type $\hat{r}$ in Period 1. The RHS gives the expected payoff. The function $\hat{i}$ is defined as the cutoff worker, which suggests that full unraveling tends to benefit low quality workers while hurting high quality workers.

Now we consider a banning policy on exploding offers. Suppose every participant holds the common belief that the market does not unravel if exploding offers are banned but fully unravels if they are allowed. Then clearly such a policy will be supported by high quality firms and workers, and opposed by firms of low or medium qualities and workers of low qualities. Similar conclusion is drawn from the experimental results in Niederle and Roth (2009). They find that while early matches are costly for the highest quality firms and workers, some lower quality firms and applicants tend to gain from them. The conclusion also explains the prevalence of exploding offers in practice and why it is sometimes difficult to achieve consensus on how to solve the unraveling problem in real markets (Niederle and Roth, 2005).

\section{Conclusion}

Many two-sided matching markets tend to unravel in time with transactions occurring earlier and earlier. Using a two-period decentralized model, this paper shows that when a market culture allows firms to make exploding offers, such unraveling is more likely to take place and lead to an unstable matching due to the lack of information in the early stages.

An excess supply of labor makes a market less vulnerable to the presence of exploding offers, while the effect of signal accuracy is ambiguous, depending on how uncertainty is resolved in a market. Therefore, although exploding offers in general tends to facilitate early transactions, it is only a necessary but not a sufficient condition for unraveling to occur. The policy regarding exploding offers should be tailored to the specific environment of interest, while taking into consideration such market characteristics as labor supply and demand and information structure. More importantly, the welfare analysis in this paper indicates that a ban towards exploding offers may benefit high quality firms and workers but hurt agents of low qualities. This suggests the need for policymakers to balance the costs and gains when addressing the unraveling problem in a market.

Admittedly, the model in this paper only provides a simple and tractable benchmark for 
the analysis of market unraveling. Some specific structures of the model may limit its ability to predict for a more general market environment. Further extensions into environments like heterogeneous preferences or asymmetric information may give us some additional insights regarding how the use of exploding offers can affect the outcome of a matching market. 


\section{Appendix: Proofs}

\section{Proof of Proposition 1}

Proof. Consider a worker who has received an early open offer from firm $f_{j}(j=1,2, \ldots, F-1)$. As long as a better firm $f_{j^{\prime}}\left(j^{\prime}>j\right)$ moves in a later period in equilibrium, she strictly prefers holding to accepting or rejecting the offer right away.

Firstly, holding is preferred to accepting. By choosing to hold but not to accept, the worker is strictly better off if she receives a better offer in a later period. If not, she is not worse off since she still has the open offer from $f_{j}$. Secondly, holding is preferred to rejecting. By choosing to hold but not to reject, the worker is not worse off if she receives a better offer in a later period; she is strictly better off otherwise.

Knowing this, the best firm $f_{F}$ strictly prefers to wait until the last period, so that all workers will stay in the market and the one of the best quality can be perfectly identified. Since no offer is accepted in Period 1, the other firms cannot make themselves better off by moving early; instead, they incur the risk of being rejected in the last period and remaining unmatched.

Hence, there always exist profitable deviations from an equilibrium with partial or full unraveling. Only an equilibrium without unraveling can sustain. It yields the assortative matching according to the true ranking of workers $\succ$, which is the unique stable matching in the current environment with strict rankings and aligned preferences.

\section{Proof of Lemma 1}

Proof. When making an offer in Period 2, every firm is indifferent because an open offer is equivalent to an exploding offer. Now consider an offer in Period 1. The best firm $f_{F}$ is still indifferent since neither an exploding offer nor an open offer will be rejected by any worker. However, for other firms, making an open offer is never the strictly best response.

First, when making an exploding offer that has to be accepted within the same period, a firm always knows whether it will be accepted. This is because there is no information asymmetry in the current setting, and the information status remains the same within a period.

Next, if an exploding offer will be accepted, making an exploding offer yields the same or a higher payoff than making an open offer. Suppose a firm makes an open offer in Period 1, there are three possible responses: (i) it is accepted right away; (ii) it is held and accepted in Period 2; (iii) it is held and rejected in Period 2. Compared to an exploding offer, the firm yields the same payoff in cases (i) and (ii), but is strictly worse off in case (iii).

Finally, if an exploding offer will be rejected, waiting yields the same or a higher payoff than making an exploding offer or an open offer. A firm never wants to make an exploding 
offer knowing it will be rejected because then it cannot make an offer to the same worker again. On the other hand, if a firm makes an open offer in Period 1, there are three possible responses: (i) it is rejected right away; (ii) it is held and accepted in Period 2; (iii) it is held and rejected in Period 2. Compared to waiting, the firm is strictly worse off in cases (i) and (iii), and is weakly worse off in case (ii).

\section{Proof of Proposition 2}

Proof. Below I first calculate the updated beliefs of firms and workers after they observe a signal in Period 1.

After observing a signal $\hat{\succ}$, posteriors on the true state are given by

$$
\begin{aligned}
\operatorname{Pr}(\hat{\succ} \mid \hat{\succ}) & =\frac{\left[\alpha+(1-\alpha) \frac{1}{W !}\right] \frac{1}{W !}}{\left[\alpha+(1-\alpha) \frac{1}{W !}\right] \frac{1}{W !}+(W !-1)\left[(1-\alpha) \frac{1}{W !}\right] \frac{1}{W !}} \\
& =\alpha+\frac{1-\alpha}{W !} .
\end{aligned}
$$

For any $\succ^{\prime} \neq \hat{\succ}$

$$
\operatorname{Pr}\left(\succ^{\prime} \mid \hat{\succ}\right)=\frac{1-\frac{\alpha W !+(1-\alpha)}{W !}}{W !-1}=\frac{1-\alpha}{W !} .
$$

Posteriors on types are given by

$$
\begin{aligned}
\operatorname{Pr}(\hat{r} \mid \hat{r}) & =\operatorname{Pr}(\hat{\succ} \mid \hat{\succ})+[(W-1) !-1] \operatorname{Pr}\left(\succ^{\prime} \mid \hat{\succ}\right) \\
& =\alpha+\frac{1-\alpha}{W}
\end{aligned}
$$

and $\forall r^{\prime} \neq \hat{r}$

$$
\operatorname{Pr}\left(r^{\prime} \mid \hat{r}\right)=\frac{1-\left(\alpha+\frac{1-\alpha}{W}\right)}{W-1}=\frac{1-\alpha}{W} .
$$

Thus, the expected quality of a type- $\hat{r}$ worker is

$$
E V(\hat{r})=\left(\alpha+\frac{1-\alpha}{W}\right) \hat{r}+\sum_{r^{\prime} \neq \hat{r}} \frac{1-\alpha}{W} r^{\prime}=\alpha \hat{r}+\frac{(1-\alpha)(W+1)}{2} .
$$

Next, consider the case where $W>F$. In an equilibrium without unraveling, no actions are taken in Period 1. In Period 2, after $\succ$ is revealed, $f_{F}$ makes an offer to $w_{W}, f_{F-1}$ to $w_{W-1}, \ldots$, and $f_{1}$ to $w_{W-F+1}$. All offers are accepted.

It is clear that workers do not have any incentive to deviate, nor does firm $f_{F}$. Given all others are playing the equilibrium strategy, a firm $f_{j}$ with $j=1,2, \ldots, F-1$ will not deviate

and make an offer to a different worker in Period 2, since it will not be accepted by a worker better than its current match $w_{j-F+W}$. I now focus on checking the deviation of $f_{j}$ in Period 
1. Such a deviation involves both sides of the market: a firm should want to make an early exploding offer to a worker who wants to accept it. So a sufficient condition for the existence of an equilibrium without unraveling is that, for each worker type $\hat{r}$, the firms whose offer would be accepted are not willing to offer.

Suppose $f_{j}$ deviates by making an early offer to a type- $\hat{r}$ worker in Period 1 , and $\hat{r} \neq \hat{r}(j)$. The offer is accepted if

$$
j \geq\left(\alpha+\frac{1-\alpha}{W}\right) \times j(\hat{r})+\frac{1-\alpha}{W} \times\left(\sum_{r^{\prime}=W-F+1}^{W} j\left(r^{\prime}\right)-j(\hat{r})\right)-\frac{1-\alpha}{W} \times 1,
$$

or equivalently,

$$
j \geq \underline{j_{1}^{A}}(\hat{r}) \equiv \alpha \times(\hat{r}-W+F)+\frac{(1-\alpha)\left(F^{2}+F-2\right)}{2 W} .
$$

The function $j_{1}^{A}(\hat{r})$ is defined as the lowest ranked firm that is accepted by type $\hat{r}$. On the other hand, if accepted, the firm is willing to make such an offer if

$$
j+W-F \leq E V(\hat{r}),
$$

or equivalently,

$$
j \leq \overline{j_{1}^{O}}(\hat{r}) \equiv \alpha \hat{r}+\frac{(1-\alpha)(W+1)}{2}-W+F .
$$

The function $\overline{j_{1}^{O}}(\hat{r})$ is defined as the highest ranked firm that wants to make an early offer to type $\hat{r}$. The sufficient condition for no deviation in this case is that for each type, there does not exist a firm that is willing to offer, and is accepted. That is, $\forall \hat{r}$, we need to have

$$
\underline{j_{1}^{A}}(\hat{r}) \geq \overline{j_{1}^{O}}(\hat{r}) \text { and } \overline{j_{1}^{O}}(\hat{r}) \geq 1
$$

or

$$
\overline{j_{1}^{O}}(\hat{r})<1
$$

which solves

$$
W \geq 2+F
$$

(10) ensures that there does not exist a $j$ such that $\underline{j_{1}^{A}}(\hat{r}) \leq j \leq \overline{j_{1}^{O}}(\hat{r})$. (11) is a boundary condition where no firms are willing to make an offer to a type- $\hat{r}$ worker. ${ }^{15}$

Now we consider the deviation of a firm $f_{j}$ to its signal-suggested type $\hat{r}(j)$. The offer is

\footnotetext{
${ }^{15}$ Another boundary case would be that a type- $\hat{r}$ worker does not accept any offer, which never holds because

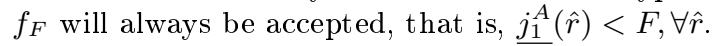


accepted if

$$
j \geq\left(\alpha+\frac{1-\alpha}{W}\right) \times j(\hat{r}(j))+\frac{1-\alpha}{W} \times\left(\sum_{r^{\prime}=W-F+1}^{W} j\left(r^{\prime}\right)-j(\hat{r}(j))\right)-\left(\alpha+\frac{1-\alpha}{W}\right),
$$

or equivalently,

$$
j \geq \underline{j_{2}^{A}} \equiv \frac{F^{2}+F-2}{2 W}-\frac{\alpha}{1-\alpha} .
$$

If accepted, the firm is willing to make such an offer if

$$
j+W-F \leq E V(\hat{r}(j))
$$

or equivalently,

$$
j \leq \overline{j_{2}^{O}} \equiv \frac{1}{2}+F-\frac{W}{2} .
$$

The sufficient condition for no deviation in this case is that there does not exist a firm willing to make an offer to its signal-suggested type, and is accepted. That is,

$$
\underline{j_{2}^{A}} \geq \overline{j_{2}^{O}} \text { and } \overline{j_{2}^{O}} \geq 1
$$

or

$$
\overline{j_{2}^{O}}<1
$$

Combining with (12), the two sufficient conditions when $W>F$ are given by (i) $W \geq 2 F$, or (ii) $F+2<W \leq 2 F-1$ and $\alpha \leq \frac{(W-F)^{2}-W+F-2}{(W-F)^{2}+W+F-2}$.

Now I move on to the case where $W \leq F$. In an equilibrium without unraveling, no actions are taken in Period 1. In Period 2, after $\succ$ is revealed, $f_{F}$ makes an offer to $w_{W}, f_{F-1}$ to $w_{W-1}, \ldots$, and $f_{F-W+1}$ to $w_{1}$. All these offers are accepted.

Suppose a firm $f_{j}$ deviates by making an early offer to its signal-suggested type $\hat{r}(j)=$ $j-F+W$. The offer is accepted if

$$
j \geq\left(\alpha+\frac{1-\alpha}{W}\right) \times j(\hat{r}(j))+\frac{1-\alpha}{W} \times\left(\sum_{r^{\prime}=1}^{W} j\left(r^{\prime}\right)-j(\hat{r}(j))\right)-\left(\alpha+\frac{1-\alpha}{W}\right),
$$

or equivalently,

$$
j \geq \underline{j_{3}^{A}} \equiv \frac{2 F-W+1}{2}-\frac{1}{W}-\frac{\alpha}{1-\alpha} .
$$

The firm wants to make such an offer if

$$
j+W-F \leq E V(\hat{r}(j))
$$


or equivalently,

$$
j \leq \overline{j_{3}^{O}} \equiv \frac{1}{2}+F-\frac{W}{2} .
$$

It is easy to show that $\underline{j_{3}^{A}}<\overline{j_{3}^{O}}$, that is, the sufficient condition for no deviation never holds for $W \leq F$.

Therefore, the equilibrium without unraveling always exists if (i) $W \geq 2 F$, or (ii) $F+2<$ $W \leq 2 F-1$ and $\alpha \leq \frac{(W-F)^{2}-W+F-2}{(W-F)^{2}+W+F-2}$.

\section{Proof of Proposition 3}

Proof. For a deviation from the equilibrium without unraveling to occur, there must exist a type of worker and a firm in Period 1 such that, the firm is willing to offer and the worker is willing to accept.

When $W>F$, for a deviation between a non-signal-suggested pair to exist, we need $\exists \hat{r}$ such that

$$
\overline{j_{1}^{O}}(\hat{r})-\underline{j_{1}^{A}}(\hat{r})>1 \text { if } \underline{j_{1}^{A}}(\hat{r}) \geq 0,
$$

or

$$
\overline{j_{1}^{O}}(\hat{r})>1 \text { if } \underline{j_{1}^{A}}(\hat{r})<0 .
$$

(21) ensures that the range between $\overline{j_{1}^{O}}(\hat{r})$ and $\underline{j_{1}^{A}}(\hat{r})$ is larger than 1 , so that there always exists an integer in between. (22) is a boundary case where $\underline{j_{1}^{A}}(\hat{r})<0$. Then we need the range to be even larger so that at least $f_{1}$ is willing to offer. ${ }^{16}$ It is easy to confirm that the two conditions above never hold.

Similarly, for a deviation between a signal-suggested pair to exist, we need

$$
\overline{j_{2}^{O}}-\underline{j_{2}^{A}}>1 \text { if } \underline{j_{2}^{A}} \geq 0
$$

or

$$
\overline{j_{2}^{O}}>1 \text { if } \underline{j_{2}^{A}}<0
$$

which solves $F<W<2 F-1$ and $\alpha>\frac{(W-F)^{2}+W+F-2}{(W-F)^{2}+3 W+F-2}$.

When $W \leq F$, for a deviation between a signal-suggested pair to exist, we need

$$
\overline{j_{3}^{O}}-\underline{j_{3}^{A}}>1 \text { if } \underline{j_{3}^{A}} \geq 0
$$

or

$$
\overline{j_{3}^{O}}>1 \text { if } \underline{j_{3}^{A}}<0
$$

which solves $W \leq F$ and $\alpha>\frac{W-1}{2 W-1}$.

\footnotetext{
${ }^{16}$ There is another boundary where $\overline{j_{1}^{O}(\hat{r})}>F$, which never holds since $f_{F}$ never wants to deviate.
} 
Therefore, the equilibrium without unraveling never exists if (i) $W \leq F$ and $\alpha>\frac{W-1}{2 W-1}$; or (ii) $F<W<2 F-1$ and $\alpha>\frac{(W-F)^{2}+W+F-2}{(W-F)^{2}+3 W+F-2}$.

\section{Proof of Proposition 4}

Proof. Consider the case where $W>F$.

In an equilibrium with full unraveling, after $\hat{\succ}$ is revealed in Period $1, f_{F}$ makes an offer to type $\hat{r}=W, f_{F-1}$ to $\hat{r}=W-1, \ldots$, and $f_{1}$ to $\hat{r}=W-F+1$. All offers are accepted. Such an equilibrium can never sustain because after Period 1 , there are $W-F$ workers left in the market. Given all the other firms move early, each firm has an incentive to deviate to Period 2, in which case it becomes the only firm left in the market and can choose the best remaining worker.

Consider the case where $W \leq F$.

In an equilibrium with full unraveling, after $\hat{\succ}$ is revealed in Period $1, f_{F}$ makes an offer to type $\hat{r}=W, f_{F-1}$ to $\hat{r}=W-1, \ldots$, and $f_{F-W+1}$ to $\hat{r}=1$. All these offers are accepted. No workers are left in the market after the first period. Therefore, a firm has no incentive to deviate as long as in its deviation, no worker would reject her current offer and become available in Period 2. That is, in the subgame after any firm's deviation, all workers still accept their offers in Period 1.

Suppose a firm $f_{j^{\prime}}$ deviates and waits until Period $2, j^{\prime}=F-W+1, F-W+2, \ldots, F$. A worker of type $\hat{r}$ would still accept her current offer if $j^{\prime} \leq j(\hat{r})$, that is, a worker would never deviate for a firm that is worse than her offer in equilibrium, which is from her signal-suggested firm $j(\hat{r})$. The binding condition for the existence of an equilibrium with full unraveling then requires type $\hat{r}=1$ not to unilaterally reject her offer in Period 1 in the deviation of $f_{F}$, that is,

$$
F-W+1 \geq \frac{1-\alpha}{2} F+\left(\alpha+\frac{1-\alpha}{2}\right)(F-W) .
$$

The RHS of (27) is the worker's payoff if she accepts her offer in equilibrium. The LHS is the worker's expected payoff if she rejects. In this case, after the first period, there are two workers $(\hat{r}=1$ and $\hat{r}=W)$ and $F-W+2$ firms $\left(f_{F}, f_{F-W+1}, f_{F-W}, f_{F-W-1}, \ldots\right.$, and $\left.f_{1}\right)$ left in the market. Type $\hat{r}=1$ is matched with $f_{F}$ if she turns out to have a higher quality than $\hat{r}=W$, and is matched with $f_{F-W}$ otherwise since $f_{F-W+1}$ is no longer available to her after the rejection. Compared to a higher type, the probability of a worker having a higher quality in the true state is given by $\frac{1-\alpha}{W !} \times \frac{W !}{2}=\frac{1-\alpha}{2}$. Equation (27) solves

$$
\alpha \geq \frac{W-2}{W}
$$

Together with the constraint $W \leq F$, an equilibrium with full unraveling exists if $W \leq F$ and $\alpha \geq \frac{W-2}{W}$. 
On the other hand, when $W \leq F$ and $\alpha<\frac{W-2}{W}$, an equilibrium with full unraveling never exists since type $\hat{r}=1$ has an incentive to reject her offer in Period 1 in the deviation of $f_{F}$, which makes $f_{F}$ strictly prefer to deviate. Combined with the fact that such an equilibrium never exists when $W>F$, we obtain the sufficient and necessary condition for the existence of an equilibrium with full unraveling: $W \leq F$ and $\alpha \geq \frac{W-2}{W}$.

\section{References}

[1] Avery, C., Fairbanks, A., And Zeckhauser, R. J. The early admissions game. Harvard University Press, 2009.

[2] Avery, C., Jolls, C., Posner, R. A., And Roth, A. E. The market for federal judicial law clerks. The University of Chicago Law Review (2001), 793-902.

[3] Echenique, F., And Pereyra, J. S. Strategic complementarities and unraveling in matching markets. Theoretical Economics 11, 1 (2016), 1-39.

[4] Fainmesser, I. P. Social networks and unraveling in labor markets. Journal of Economic Theory 148, 1 (2013), 64-103.

[5] Fréchette, G. R., Roth, A. E., And Ünver, M. U. Unraveling yields inefficient matchings: evidence from post-season college football bowls. The RAND Journal of Economics 38, 4 (2007), 967-982.

[6] HaŁaburda, H. Unravelling in two-sided matching markets and similarity of preferences. Games and Economic Behavior 69, 2 (2010), 365-393.

[7] Haruvy, E., Roth, A. E., And Ünver, M. U. The dynamics of law clerk matching: An experimental and computational investigation of proposals for reform of the market. Journal of Economic Dynamics and Control 30, 3 (2006), 457-486.

[8] Kagel, J. H., And Roth, A. E. The dynamics of reorganization in matching markets: A laboratory experiment motivated by a natural experiment. The Quarterly Journal of Economics 115, 1 (2000), 201-235.

[9] Kemeny, J. G. Mathematics without numbers. Daedalus 88, 4 (1959), 577-591.

[10] Kendall, M. G. A new measure of rank correlation. Biometrika (1938).

[11] LEE, S.-H. Jumping the curse: Early contracting with private information in university admissions*. International Economic Review 50, 1 (2009), 1-38.

[12] Li, H., And Rosen, S. Unraveling in matching markets. American Economic Review 88, 3 (1998), 371-87. 
[13] Li, H., And Suen, W. Risk sharing, sorting, and early contracting. Journal of Political Economy 108, 5 (2000), 1058-1091.

[14] Li, H., And Suen, W. Self-fulfilling early-contracting rush*. International Economic Review 45, 1 (2004), 301-324.

[15] McKinney, C. N., Niederle, M., and Roth, A. E. The collapse of a medical labor clearinghouse (and why such failures are rare). American Economic Review 95, 3 (2005), 878-889.

[16] Mongell, S., And Roth, A. E. Sorority rush as a two-sided matching mechanism. The American Economic Review (1991), 441-464.

[17] Niederle, M., And Roth, A. E. Unraveling reduces mobility in a labor market: Gastroenterology with and without a centralized match. Journal of political Economy 111, 6 (2003), 1342-1352.

[18] Niederle, M., And Roth, A. E. The gastroenterology fellowship market: Should there be a match? American Economic Review (2005), 372-375.

[19] Niederle, M., And Roth, A. E. Market culture: How rules governing exploding offers affect market performance. American Economic Journal: Microeconomics 1, 2 (2009), $199-219$.

[20] Niederle, M., Roth, A. E., And Ünver, M. U. Unraveling results from comparable demand and supply: An experimental investigation. Games 4, 2 (2013), 243-282.

[21] Roth, A. E. The evolution of the labor market for medical interns and residents: a case study in game theory. The Journal of Political Economy 92, 6 (1984), 991.

[22] Roth, A. E. A natural experiment in the organization of entry-level labor markets: regional markets for new physicians and surgeons in the united kingdom. The American economic review (1991), 415-440.

[23] Roth, A. E., And Xing, X. Jumping the gun: Imperfections and institutions related to the timing of market transactions. The American Economic Review (1994), 992-1044.

[24] Roth, A. E., And Xing, X. Turnaround time and bottlenecks in market clearing: Decentralized matching in the market for clinical psychologists. Journal of Political Economy 105, 2 (1997), 284-329.

[25] Suen, W. A competitive theory of equilibrium and disequilibrium unravelling in twosided matching. The Rand journal of economics (2000), 101-120. 
[26] Ünver, M. U. Backward unraveling over time: The evolution of strategic behavior in the entry level british medical labor markets. Journal of Economic dynamics and control 25, 6 (2001), 1039-1080.

[27] Ünver, M. U. On the survival of some unstable two-sided matching mechanisms. International Journal of Game Theory 33, 2 (2005), 239-254. 